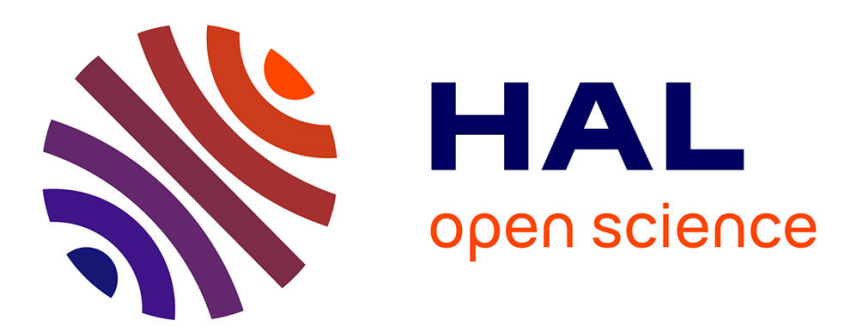

\title{
Evaluation of gradient operators for hyperspectral image processing
}

Hilda Deborah, Noël Richard, Jon Hardeberg, Christine Fernandez-Maloigne

\section{To cite this version:}

Hilda Deborah, Noël Richard, Jon Hardeberg, Christine Fernandez-Maloigne. Evaluation of gradient operators for hyperspectral image processing. Color and Imaging Conference, Volume 2017, Number 25, Sep 2017, Lillehammer, Norway. hal-02407240

\section{HAL Id: hal-02407240 \\ https://hal.science/hal-02407240}

Submitted on 12 Dec 2019

HAL is a multi-disciplinary open access archive for the deposit and dissemination of scientific research documents, whether they are published or not. The documents may come from teaching and research institutions in France or abroad, or from public or private research centers.
L'archive ouverte pluridisciplinaire HAL, est destinée au dépôt et à la diffusion de documents scientifiques de niveau recherche, publiés ou non, émanant des établissements d'enseignement et de recherche français ou étrangers, des laboratoires publics ou privés. 


\title{
Evaluation of gradient operators for hyperspectral image processing
}

\author{
Hilda Deborah; Colourlab, Dept. of Computer Science, Norwegian University of Science \& Technology; Gjøvik, Norway \\ Noël Richard; XLIM Laboratory, UMR CNRS 7252, University of Poitiers; Futuroscope Cedex, France \\ Jon Y. Hardeberg; Colourlab, Dept. of Computer Science, Norwegian University of Science \& Technology; Gjøvik, Norway \\ Christine Fernandez-Maloigne; XLIM Laboratory, UMR CNRS 7252, University of Poitiers; Futuroscope Cedex, France
}

\begin{abstract}
Gradient is an important image processing tool allowing to carry out edge detection, segmentation, and texture analysis. It is expected to provide significantly more accurate and complete information when combined with the hyperspectral imaging technology. And such gain in accuracy justifies the cost and complexity of a hyperspectral acquisition. But how to measure accuracy? This article presents our first study in the accuracy assessment of hyperspectral gradient, where an assessment protocol based on a basic edge detection task is proposed. This protocol is then used to evaluate the full-band gradient approaches, where the results suggest to improve the protocol to include more complexity.
\end{abstract}

\section{Introduction}

The gradient of an image captures directional changes of pixel values in the spatial coordinates of the image. For intensity images, gradient is defined only by its norm and spatial direction, obtained from the derivative forms of a discrete image. For hyperspectral images, as for color images, the direction in the spectral space must also be considered in order to completely define the hyperspectral gradient. But how should the derivation of spectral images be developed?

Hyperspectral image processing is employed for its potential in providing highly accurate results. However, to preserve the acquired image quality in the subsequent processing chain, each processing step must be carried out in a full-band manner. Thus, data reduction techniques such as PCA are to be avoided. The full-band approach to gradient extraction requires gradient to be defined with regards to the definition of a spectral function, allowing to establish the derivative form of a hyperspectral image.

In [13], it was shown that a spectrum must be considered as a continuous digitalized function. The provided demonstration followed protocols and results previously obtained in [5], which had presented the limitations of approaches that consider a spectrum as a vector or probability density function. Afterwards, the term spectrum will be replaced by its correct definition, i.e., spectral function. These achievements have allowed defining a metrologically valid spectral difference measure, subsequently enabling the construction of a full-band spectral ordering relation that satisfies metrological constraints [6]. The development was continued and a full-band spectral mathematical morphology was obtained, including a proposal of a spectral Beucher gradient in [4]. The work aimed to address the question of whether there is an interest to the complexity of a full-band processing, whether it preserves and valorizes the accuracy of an acquired hyperspectral image.

In this paper, the extensions of several known image gradi- ent operators to the spectral domain are shown. Aiming to measure their accuracy, an edge detection-based assessment protocol is developed. This includes the use of an existing criterion and a proposal of a basic spectral dataset specifically developed for gradient assessment task.

\section{Image gradient operators}

The concept of edge in an image can be expressed either in the spatial or frequency domains. Focusing on the former, in this work we associate the identification of an edge to the computation of gradients and other derivative forms of an image. Table 1 provides the mathematical notations frequently used in this article.

\section{From graylevel to multivariate gradients}

In the continuous domain, the derivative of a onedimensional signal $t(x)$ can be expressed as follows.

$$
\nabla t(x) \equiv \lim _{\Delta x \rightarrow 0} \frac{t(x+\Delta x)-t(x)}{\Delta x}
$$

Its extension to the two-dimensional case is obtained below, by partial derivations of each axis.

$$
\nabla f_{w}(x, y)=\left[\begin{array}{l}
g_{x w} \\
g_{y w}
\end{array}\right]=\left[\begin{array}{l}
\partial f_{w}(x, y) / \partial x \\
\partial f_{w}(x, y) / \partial y
\end{array}\right]
$$

With this expression, several image derivatives were proposed for grayscale images, e.g., Prewitt and Sobel operators. They combine a directional gradient detection and a low-pass filter which reduces the sensitivity of gradient computation to noise. Their local gradients are then obtained using the norm and direction of gradient as follows.

$$
\left|\nabla f_{w}(x, y)\right|=\sqrt{g_{x w}^{2}+g_{y w}^{2}},\left\llcorner\nabla f_{w}(x, y)=\operatorname{atan}\left[\frac{g_{y w}}{g_{x w}}\right]\right.
$$

These gradients were further extended to the multivariate domain, essentially in the context of color images $[11,18]$. However, the extension is more complex when it comes to hyperspectral images. As illustrated in Fig. 1, a pixel value is not a three-component color value but a function of wavelength $\mathcal{S}(\lambda)$. The basic strategies approximate multivariate gradient through a marginal approach, followed by combining the marginal results with a maximum function in (4) or weighted sum [16].

$$
\begin{aligned}
& |\nabla f(x, y)|=\max _{w \in\left[0, n_{W}-1\right]}\left\{\left|\nabla f_{w}(x, y)\right|\right\} \\
& \left\llcorner\nabla f(x, y)=\underset{w \in\left[0, n_{W}-1\right]}{\operatorname{argmax}}\left\{\left\llcorner\nabla f_{w}(x, y)\right\}\right.\right.
\end{aligned}
$$


Table 1: Mathematical notations

\begin{tabular}{|c|c|}
\hline$f(x, y)$ & $\begin{array}{l}\text { A digital image as a function of two dimensional } \\
\text { spatial coordinates } x, y \in \mathbb{Z} \text {; A spectral image is } \\
\text { expressed as } f(x, y)=S\end{array}$ \\
\hline $\mathcal{S}(\lambda)$ & $\begin{array}{l}\text { A continuous expression of spectrum as a function } \\
\text { of wavelength } \lambda\end{array}$ \\
\hline $\mathbf{S}$ & $\begin{array}{l}\text { A sampled/ discrete expression of spectral func- } \\
\text { tion } \mathcal{S} \text {, where } \mathbf{S}=\left\{s_{w}, w \in\left[0, n_{w}-1\right]\right\}\end{array}$ \\
\hline$n_{w}, w$ & $\begin{array}{l}\text { Number of sampled spectral bands or wavelengths } \\
\lambda \text { and any arbitrary one }\end{array}$ \\
\hline$f_{w}(p)$ & $\begin{array}{l}\text { An intensity image corresponding to the } w \text {-th spec- } \\
\text { tral band }\end{array}$ \\
\hline$s_{w}$ & A spectral measure at a sampled spectral band $w$ \\
\hline $\begin{array}{l}\nabla f,|\nabla f| \\
\llcorner\nabla f\end{array}$ & $\begin{array}{l}\text { Gradient of image } f \text {, its norm, and its direction, } \\
\text { respectively }\end{array}$ \\
\hline$g_{x}, g_{y}$ & Partial derivation of an image in each of its axes \\
\hline$\Lambda_{+}, \Lambda_{-}$ & Eigenvalues in the context of Di Zenzo gradient \\
\hline$B$ & An arbitrary structuring element centered in $(x, y)$ \\
\hline$\delta_{B}, \varepsilon_{B}$ & $\begin{array}{l}\text { Dilation and erosion, respectively, relative to flat } \\
\text { structuring element } B\end{array}$ \\
\hline$O(\mathcal{S})$ & Ordering relation with spectral function $\mathcal{S}$ as input \\
\hline$d\left(\mathcal{S}_{1}, \mathcal{S}_{2}\right)$ & $\begin{array}{l}\text { Difference function between two arbitrary spectral } \\
\text { functions } \mathcal{S}_{1} \text { and } \mathcal{S}_{2}\end{array}$ \\
\hline $\begin{array}{l}\mathcal{S}^{-\infty} \\
\mathcal{S}^{+\infty}\end{array}$ & $\begin{array}{l}\text { Minimum and maximum convergence coordi- } \\
\text { nates, respectively }\end{array}$ \\
\hline$\vee, \wedge$ & Maximum and minimum operators, respectively \\
\hline$\mu, \sigma$ & Average and standard deviation, respectively \\
\hline
\end{tabular}

The problem with a marginal approach lies in the fact that it does not preserve metrological constraints and is less sensitive to gradient coherence between the spectral bands [7]. With this consideration, Di Zenzo [7] and, later, Cumani [3] proposed a way to extend gradient to the multivariate domain. Starting by expressing the square of a minimal displacement around a spatial coordinate $(x, y)$, the quadratic expression below was proposed.

$$
\begin{aligned}
& \partial f_{w}(x, y)^{2}=a . \partial x^{2}+2 b . \partial x \partial y+c . \partial y^{2} \text { where } \\
& a=\sum_{w=0}^{n_{w}-1}\left(\frac{\partial f_{w}(x, y)}{\partial x}\right)^{2}, c=\sum_{w=0}^{n_{w}-1}\left(\frac{\partial f_{w}(x, y)}{\partial y}\right)^{2}, \\
& \text { and } b=\sum_{w=0}^{n_{w}-1} \frac{\partial f_{w}(x, y)}{\partial x} \cdot \frac{\partial f_{w}(x, y)}{\partial y}
\end{aligned}
$$

Its gradient norm is then computed by, first, obtaining the extrema of this quadratic form, i.e., eigenvalues $\Lambda_{+}$and $\Lambda_{-}$in (6). In this expression, the term $\sqrt{(a-c)^{2}+4 b^{2}}$ expresses the coherence between marginal gradient directions [3, 7].

$$
\Lambda_{ \pm}=\frac{1}{2}\left(a+c \pm \sqrt{(a-c)^{2}+4 b^{2}}\right)
$$

Then, Di Zenzo and Cumani estimated the 'edge strength' locally at point $(x, y)$ by $\sqrt{\Lambda_{+}}$, thus extending the one-dimensional case since $\Lambda_{-} \equiv 0$ for grayscale images. But, Sapiro [15] expressed that "the 'strength' of an edge in multi-valued case is not given simply by the rate of maximal change $\Lambda_{+}$, but by how $\Lambda_{+}$ compares to $\Lambda_{-}$" and, thus, further proposing the gradient norm approximation as a function of $\left(\Lambda_{+}-\Lambda_{-}\right)$.
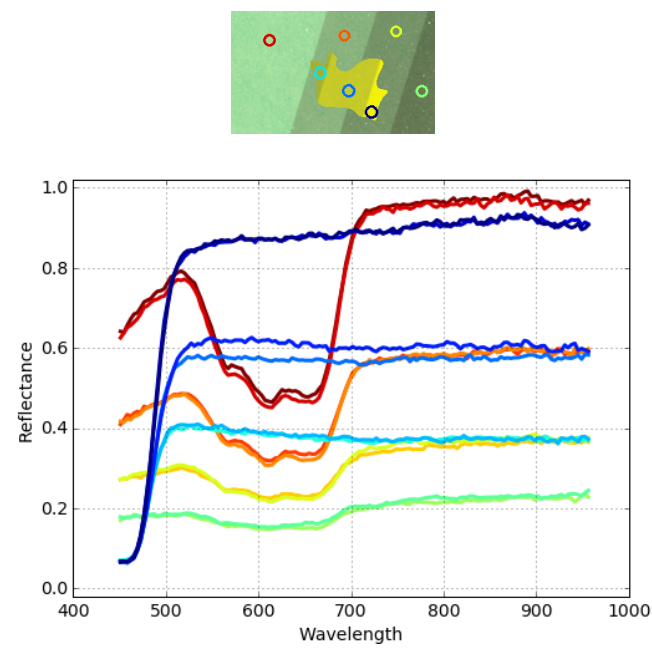

Figure 1. Illustration of pixel value in a hyperspectral image as a function over the wavelength $\mathcal{S}(\lambda)$. Spectral functions obtained within the respective colored circles in the top figure are plotted in the bottom figure.

In all of the aforementioned expressions, the problem of multivariate gradients have been formulated in a theoretical domain, where an image is considered in a vector space and, thus, its channels considered independent. In the case of hyperspectral images, these assumptions are not satisfied [5, 13]. This calls for defining a suitable approach, even if the obtained results of these existing approaches can be reasonably good in a number of cases.

\section{Existing spectral morphological gradients}

Limitations of continuous approaches to the gradient extraction of discrete images was expressed in [14]. Starting from the definition of a morphological gradient in a continuous space, its equivalence to a continuous derivative form was shown and further extended to the discrete domain, see (7). The morphological gradient is also known as the Beucher gradient.

$$
\nabla f=\delta_{B}(f)-\varepsilon_{B}(f)
$$

The extension of the Beucher gradient to the hyperspectral domain is not straightforward. It is necessary to develop an ordering relation that is valid for the spectral domain. To avoid this complex question, a marginal approach has been employed, by weighting or aggregating gradients obtained individually for each channel [9]. An alternative approach can be found in [8]. It reduces (7) to the local extraction of a maximum distance between pixels $\left(x_{i}, y_{i}\right)$ in the spatial neighborhood of a structuring element $\mathcal{P}_{B}$, see (8). However, this approach suffers from the same theoretical limitations expressed by Di Zenzo [7], i.e., the inability to account for channel coherence in the gradient extraction.

$$
\left.\nabla f(x, y)=\max _{\forall\left(x_{i}, y_{i}\right) \in \mathcal{P}_{B}}\left\{\| f\left(x_{1}, y_{1}\right), f\left(x_{2}, y_{2}\right)\right) \|_{m}\right\}
$$

\section{A full-band approach to spectral gradients}

The main challenge in extending mathematical morphology framework to the multivariate domain lies in defining a suitable ordering relation, especially as there are multitudes of them $[1,17]$. In the particular case of hyperspectral images, more 
constraints are required. First, there is the necessity to preserve accuracy and, thus, maintaining the metrological properties of spectral gradient. The latter constraint demands preserving the physical sense of spectral measures since they are acquired by spectral sensors. Then, taking the constraints into account, an ordering relation must be constructed from a valid spectral distance function. Finally, when these are accounted for, metrological assessment of morphological operators will become possible [4].

\section{Distance-based spectral ordering relation}

The notions of maximum and minimum, which are central in morphological processing, are dependent on the processing goals. Thus, they must be defined through convergence coordinates, either in a supervised or unsupervised manner. The ordering relation in (9) was defined within the supervised context, where $\mathcal{S}^{-\infty}$ and $\mathcal{S}^{+\infty}$ are minimum and maximum convergence coordinates, respectively [4].

$$
\begin{aligned}
& \mathcal{S}_{1} \preceq \mathcal{S}_{2} \Leftrightarrow\left\{\begin{array}{c}
O_{R}^{-}\left(\mathcal{S}_{1}\right)>O_{R}^{-}\left(\mathcal{S}_{2}\right) \text { or } \\
O_{R}^{-}\left(\mathcal{S}_{1}\right)=O_{R}^{-}\left(\mathcal{S}_{2}\right), O_{A}\left(\mathcal{S}_{1}\right)<O_{A}\left(\mathcal{S}_{2}\right)
\end{array}\right. \\
& \mathcal{S}_{1} \succeq \mathcal{S}_{2} \Leftrightarrow\left\{\begin{array}{c}
O_{R}^{+}\left(\mathcal{S}_{1}\right)>O_{R}^{+}\left(\mathcal{S}_{2}\right) \text { or } \\
O_{R}^{+}\left(\mathcal{S}_{1}\right)=O_{R}^{+}\left(\mathcal{S}_{2}\right), O_{A}\left(\mathcal{S}_{1}\right)>O_{A}\left(\mathcal{S}_{2}\right)
\end{array}\right. \\
& \text { where } O_{R}^{-}(\mathcal{S})=\frac{d\left(\mathcal{S}, \mathcal{S}^{+\infty}\right)}{d\left(\mathcal{S}, \mathcal{S}^{-\infty}\right)}, O_{R}^{+}(\mathcal{S})=\frac{d\left(\mathcal{S}, \mathcal{S}^{-\infty}\right)}{d\left(\mathcal{S}, \mathcal{S}^{+\infty}\right)}
\end{aligned}
$$

In this paper, both $\mathcal{S}^{-\infty}$ and $\mathcal{S}^{+\infty}$ are defined by a theoretical black and white spectral functions where values at each spectral band are the minimum and maximum of the value space, respectively. Constraints expressed in (9) are needed to enable total ordering property and to preserve morphological properties, i.e., duality, idempotency, and the physical sense of complementary.

Since in (9) the ordering relation combines measures of distances $d$ to the convergence coordinates, the key question is which distance function to use. A spectral function $\mathcal{S}$ is not a vector in the Euclidean space [5]. Consequently, distance functions such as the Euclidean distance and spectral angle [10] cannot ensure validity of results as required by metrology. A suitable spectral difference function was proposed [13], i.e., spectral KullbackLeibler pseudo-divergence (KLPD) in (10). It is composed of two independent measures of color and intensity differences, $\Delta C$ and $\Delta I$, between two continuous spectral functions.

$$
\begin{aligned}
d_{\mathrm{KLPD}}\left(\mathcal{S}_{1}, \mathcal{S}_{2}\right) & =\Delta C\left(\mathcal{S}_{1}, \mathcal{S}_{2}\right)+\Delta I\left(\mathcal{S}_{1}, \mathcal{S}_{2}\right) \\
\Delta C\left(\mathcal{S}_{1}, \mathcal{S}_{2}\right) & =k_{1} \cdot \operatorname{KL}\left(\overline{\mathcal{S}}_{1}, \overline{\mathcal{S}}_{2}\right)+k_{2} \cdot \operatorname{KL}\left(\overline{\mathcal{S}}_{2}, \overline{\mathcal{S}}_{1}\right) \\
\Delta I\left(\mathcal{S}_{1}, \mathcal{S}_{2}\right) & =\left(k_{1}-k_{2}\right) \log \left(\frac{k_{1}}{k_{2}}\right)
\end{aligned}
$$

$\overline{\mathbf{S}}, k$, and KL in (11) are normalized spectral function, its normalizing factor, and Kullback-Leibler divergence function.

$$
\begin{aligned}
& K L\left(\overline{\mathcal{S}}_{1}, \overline{\mathcal{S}}_{2}\right)=\int_{\lambda_{\min }}^{\lambda_{\max }} \overline{\mathcal{S}}_{1}(\lambda) \cdot \ln \frac{\overline{\mathcal{S}}_{1}(\lambda)}{\overline{\mathcal{S}}_{2}(\lambda)} d \lambda, \\
& k=\int_{\lambda_{\min }}^{\lambda_{\max }} \mathcal{S}(\lambda) d \lambda, \overline{\mathcal{S}} \approx \overline{\mathbf{S}}=\left\{\frac{s_{w}}{k}, \forall w \in\left[0, n_{w}-1\right]\right\}
\end{aligned}
$$

\section{Spectral Beucher gradient}

Since there is no valid addition operation for hyperspectral data, the morphological operators are limited to the use of flat structuring elements* ${ }^{*}$ Thus, dilation and erosion are defined as

$$
\begin{aligned}
& \delta_{B}(f(x, y))=\bigvee_{\left(x_{i}, y_{i}\right) \in \mathcal{P}_{B}} \mathcal{S}\left(x_{i}, y_{i}\right) \\
& \varepsilon_{B}(f(x, y))=\bigwedge_{\left(x_{i}, y_{i}\right) \in \mathcal{P}_{B}} \mathcal{S}\left(x_{i}, y_{i}\right) .
\end{aligned}
$$

The remaining task to extend the Beucher gradient to the spectral domain is to replace the subtraction operation in (7) by KLPD function, thus obtaining following expression.

$$
\nabla f=d_{K L P D}\left(\delta_{B}(f), \varepsilon_{B}(f)\right)
$$

Recalling from (10), that KLPD is decomposable into independent measures of color and intensity differences, the spectral Beucher gradient can also be decomposed into color and intensity components by replacing $d_{K L P D}$ with $\Delta C$ or $\Delta I$. In addition to embedding the difference function and ordering relation suitable for the spectral domain, this gradient is the first full-band one that is able to define the gradient norm, spatial- and spectral-direction for hyperspectral images. This proposal of the spectral Beucher gradient can be found in [4].

\section{Evaluation protocol of spectral gradients}

Image gradients can be used for edge detection tasks or for a more advanced purpose of texture analysis. But before applying spectral gradients to real application tasks using, e.g., natural images, it is important to measure the performance and validate these gradients with an increasing level of image and task complexities. In the following, a protocol of spectral gradient assessment is proposed, by means of a more basic task of edge detection. An image dataset serving this purpose is thus required, particularly one with ground truth information available. The protocol will then be used to assess the performance of three gradient operators for spectral images, i.e., extended Sobel combined using a maximum function (Max-Sobel), extended Di Zenzo-Sapiro operator, and the spectral Beucher gradient.

\section{Image datasets}

Very few hyperspectral image datasets are available and even fewer are suitable for the accuracy assessment of edge detection algorithms. Ground truth information of the available datasets is typically human-annotated segmentation results of remote sensing images [2]. In the context of cultural heritage applications, spatial resolution of the data is significantly higher. Thus, optical mixing characteristics induced by acquisition distance in the context of remote sensing is reduced. Finally, there is a need for a new spectral dataset specifically developed for assessing the accuracy of edge detection in spectral images.

Using hyperspectral images acquired from pigment patches and a painting from our repository, a dataset is created following the protocol shown in Fig. 2. Each image in the dataset is created by selecting image subsets using one of the shown object templates as the foreground object. The extracted object is then

\footnotetext{
${ }^{*}$ In the context of Sobel and Di Zenzo filters, structuring element is equivalent to the filtering window.
} 


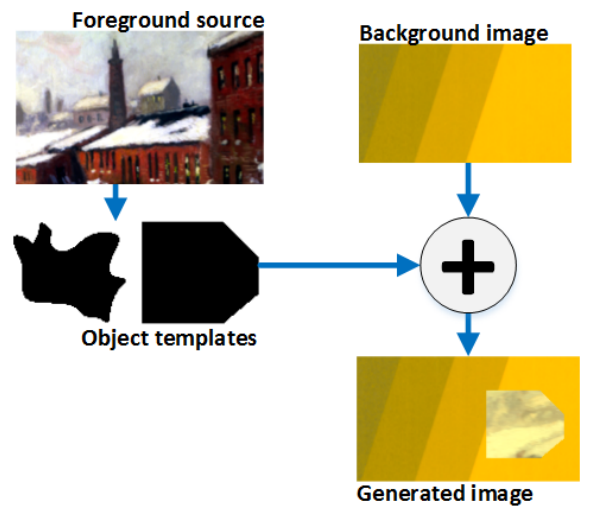

Figure 2. Image dataset generation protocol. Using an object template (out of two), a target image is generated by patching an object whose content is obtained from the foreground image source to the background image source.

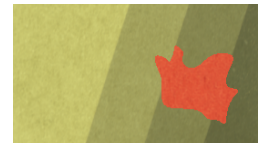

(a) KKA

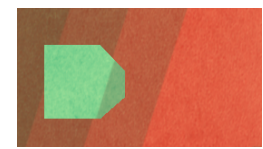

(b) KKB

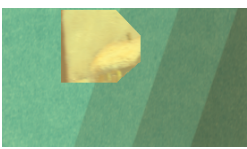

(c) KFR
Figure 3. Three image targets for the gradient computation, each an example from the respective dataset. Each dataset consists of 112 image targets. Note that all images are generated by mixing contents obtained from different hyperspectral images of real physical objects.

patched onto another image that will play the role of background content. The use of these object templates allow to obtain nonambiguous edge locations in the generated image. Selecting to work with datasets of varying but relatively low content complexity, three datasets are generated. In each case, the background part of the image comes from images of pigment patches. The increasing complexity comes from the selected foreground object content. They are obtained from homogeneous (KKA dataset) and heterogeneous (KKB dataset) regions of pigment patches which are different from the chosen background pigment. The last dataset is generated by taking the foreground object from a hyperspectral image of a painting (KFR dataset). Finally, we obtain 3 datasets each containing 112 images, see examples in Fig. 3.

\section{Evaluation criteria}

Each target image in the dataset comes with a ground truth image, showing the exact location of the foreground object and its edge. With this, an evaluation criteria originally developed for edge map quality measure will be employed [12]. Considering the evaluation of edge location based on edge presence and localization, the criteria is expressed below.

$$
\begin{aligned}
& E=1-\frac{E_{F P}+E_{F N}}{2}, \text { where } \\
& E_{F P}=\frac{1}{n_{f}-n_{O}} \sum_{i=1}^{n_{F P}}\left(1-\frac{1}{1+\alpha d_{F P_{i}}^{2}}\right), \text { and } \\
& E_{F N}=\frac{1}{n_{O}} \sum_{i=1}^{n_{F N}}\left(1-\frac{1}{1+\alpha d_{F N_{i}}^{2}}\right)
\end{aligned}
$$

$n_{f}, n_{O}, n_{F P}$, and $n_{F N}$ are image target size, number of edge pixels in the ground truth edge image, number of false positive, and of false negative pixels in the edge image under evaluation, respectively. $d_{F P_{i}}$ is the distance between $i$-th false positive edge pixel and the nearest ideal edge pixel, $d_{F N_{i}}$ is the distance between $i$-th false negative edge pixel and the nearest correctly detected edge pixel, and $\alpha$ is a penalty score set to $1 / 9$ as suggested by [19]. The evaluation scores range between 0 and 1, with 1 indicating a perfect match. Note that the criteria in (14) was developed for edge detection performance assessment, thus requiring to binarize the obtained gradient images. In this work, binarization is carried out by means of threshold selection maximizing the said criteria.

\section{Results and discussion}

Performance evaluation of the spectral Beucher, Di ZenzoSapiro, and Max-Sobel gradient operators for each group of image targets is provided in Fig. 4. Their average and standard deviation performance scores are also provided in Table 2. For KKA and KKB datasets, Max-Sobel outperforms the spectral Beucher and Di Zenzo-Sapiro gradients. This is as expected since a spectral band characterizing the foreground object can typically be found for these images. As an illustration, spectral functions obtained from both background and foreground contents of a red pigment image (Fig. 5) are given in Fig. 6. For this image, differences between the spectral functions of background and foreground parts can be summarized by the small peak at approximately $540 \mathrm{~nm}$. Performance of Max-Sobel decreases and starts to be comparable to other gradient operators when the more complex KFR dataset is employed. However, performances of these gradient operators are not significantly different. Their average scores are very close to 1.0 and standard deviations approaching 0 .

Table 2: Average and standard deviation of performances of the spectral Beucher, Di Zenzo-Sapiro, and Max-Sobel.

\begin{tabular}{|c|c|c|c|c|c|c|}
\hline \multirow{2}{*}{$\begin{array}{c}\text { Data- } \\
\text { set }\end{array}$} & \multicolumn{2}{|c|}{$\begin{array}{c}\text { Spectral } \\
\text { Beucher }\end{array}$} & \multicolumn{2}{c|}{$\begin{array}{c}\text { Di Zenzo- } \\
\text { Sapiro }\end{array}$} & \multicolumn{2}{c|}{ Max-Sobel } \\
\cline { 2 - 7 } & $\mu$ & $\sigma$ & $\mu$ & $\sigma$ & $\mu$ & $\sigma$ \\
\hline KKA & 0.998 & 0.006 & 0.998 & 0.008 & 0.999 & 0.006 \\
KKB & 0.996 & 0.014 & 0.995 & 0.016 & 0.997 & 0.007 \\
KFR & 0.987 & 0.012 & 0.983 & 0.015 & 0.989 & 0.012 \\
\hline
\end{tabular}

Observe also the results shown in Fig. 5, where each image target is causing the lowest score for the spectral Beucher (Fig. 4). For KKA and KKB, it is expected that when the background and foreground contents are of very similar colors, the gradient operators will be challenged. If the spectral content of the image is as illustrated in Fig. 6, where the characterizing information is located in very few spectral bands around $540 \mathrm{~nm}$ and also of very low intensity, slight variations in the background would easily confuse the gradient operators. Especially since the background and foreground reflectance spectral functions are of very similar overall intensity. It is due to this that KKA and KKB results in Fig. 6 are rather noisy. But nevertheless, visually, there is no significant differences between all gradient images of the same image in Fig. 6 . Finally, these results suggest that there is a need to improve the proposed image datasets. They further indicate that for each pixel in the employed datasets, there is almost always one spectral band allowing to characterize the different objects. Due to this, Max- 

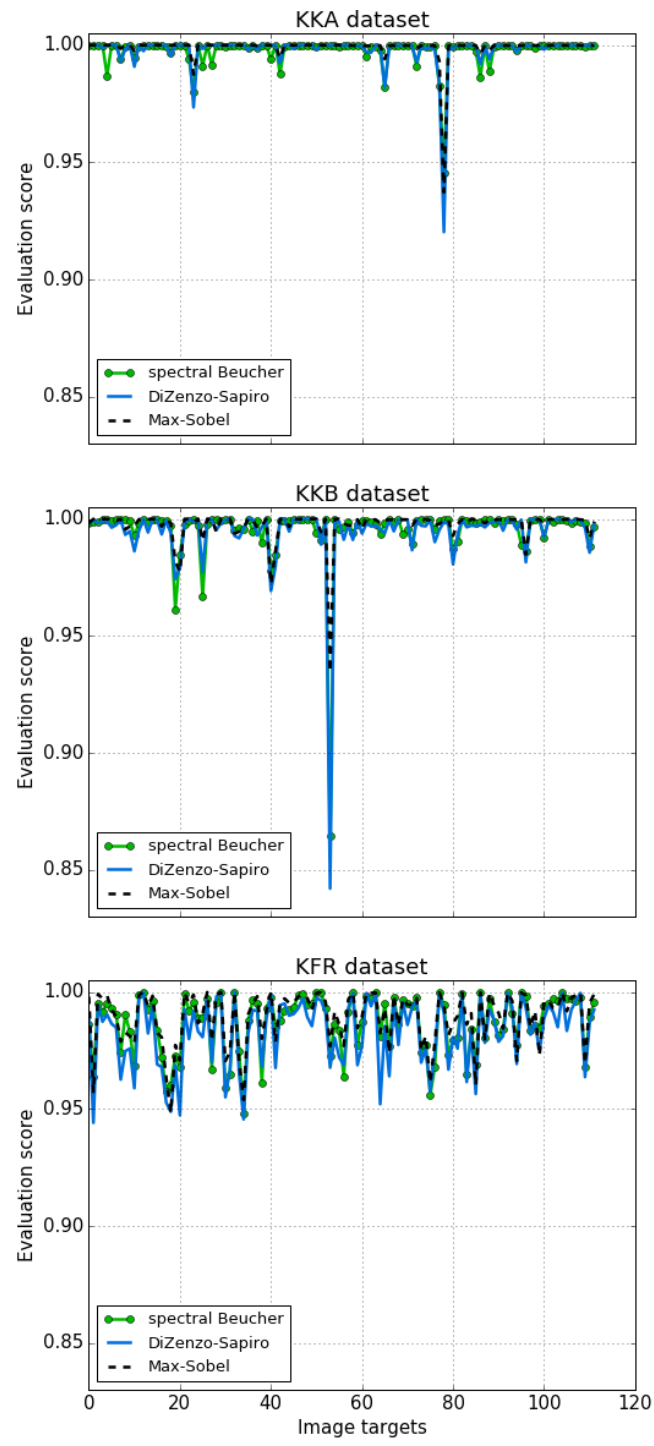

Figure 4. Evaluation scores of each gradient operator for each image target in the respective dataset. Better performance is achieved when the score is approaching 1. Nevertheless, there is no significant difference between the operator performances. Note the identical $x$-axes for all sub-figures.

Sobel operator is able to perform well but at the same time the full-band approach of the spectral Beucher is not valorized.

\section{Conclusion}

Gradient processing is at the core of many image processing and analysis tools, from image segmentation to texture analysis. To preserve the high spectral sampling and metrological properties of a hyperspectral image, this work focuses on full-band gradient detection for hyperspectral images. Three operators were presented. The first operator works in a marginal way, searching per-channel maximum gradient, but unable to define gradient norm and gradient spatial- and spectral-directions. The second operator extends the Di Zenzo construction by considering a spectral function as a vector, without the ability to process spectral gradient direction. The third one extends the Beucher gradient to
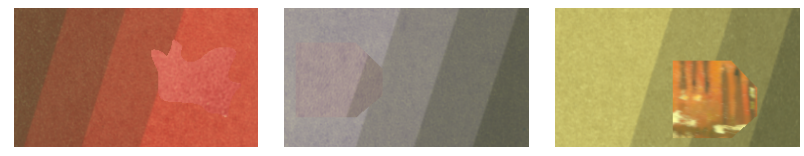

Original images, left to right: KKA, KKB, KFR
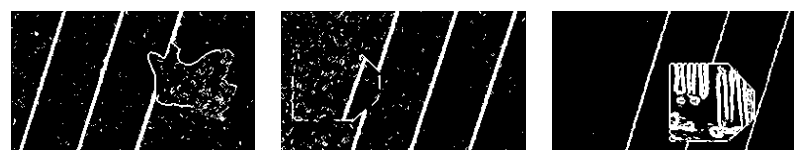

The spectral Beucher gradients
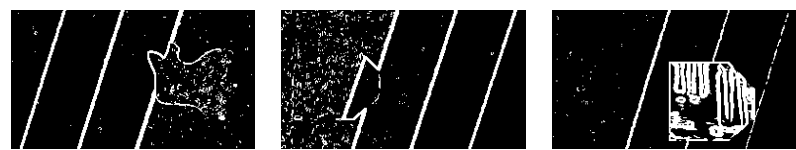

Di Zenzo-Sapiro gradients
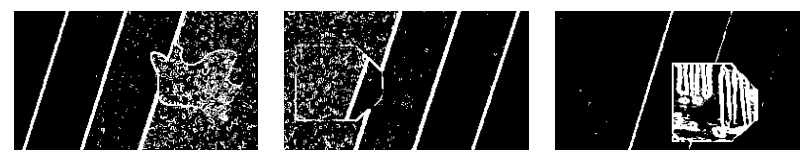

Max-Sobel gradients

Figure 5. Target images of $K K A, K K B$, and KFR datasets yielding the lowest scores for the spectral Beucher in Fig. 4. Given in the same column are their corresponding gradient images obtained by the 3 operators.

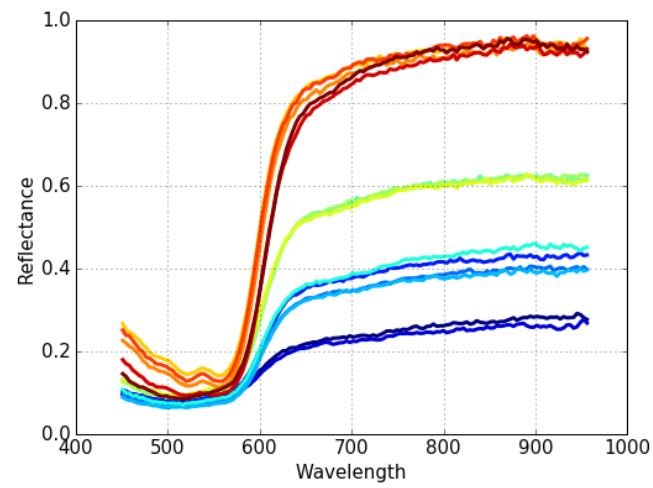

Figure 6. Spectral reflectance functions of the red pigment image in Fig. 5. Spectral functions corresponding to the foreground object present a small peak around $540 \mathrm{~nm}$, which does not appear in those of the background.

the hyperspectral domain, with the ability to process 3 gradient features, i.e., the norm and spatial- and spectral-directions.

Since there is no hyperspectral database dedicated to the accuracy assessment of gradient detection, a hyperspectral dataset of increasing complexity was proposed. With background content obtained from relatively homogeneous pigment patches, the complexity of each image is determined by the selected foreground object. Aiming to test the performance of spectral gradient operators on a basic edge detection task, this image dataset is designed to have a rather low complexity. Dataset with higher content complexity will be needed for a more complex task of texture analysis.

Performances of the three gradient operators were found to be very good, with scores closely approaching 1 in a range of 0 1. It was also observed that they decrease as spectral complexity 
of the content increases. The marginal approach obtained slightly better scores, indicating that there is always a single characteristic spectral band for each image in the dataset. These results suggest the limitations of the proposed dataset and the necessity to improve it in order to discover the limitation of each gradient operator. Nevertheless, in a more generic manner, the spectral Beucher is the only one able to provide the 3 gradient features. Due to this, it can be selected for a metrological gradient extraction.

\section{Acknowledgment}

This work is partly supported by PatAttriMetro project of the French ERDF NUMERIC and the HyPerCept project funded by the Research Council of Norway.

\section{References}

[1] V. Barnett. The ordering of multivariate data. Journal of the Royal Statistical Society. Series A (General), 139(3):318355, 1976.

[2] Computational Intelligence Group, University of the Basque Country. Hyperspectral remote sensing scenes. https:// goo.gl/668e61. Retrieved Nov 2016.

[3] A. Cumani. Edge detection in multispectral images. Graphical Models Image Processing, 53(1):40-51, 1991.

[4] H. Deborah. Towards Spectral Mathematical Morphology. $\mathrm{PhD}$ thesis, Norwegian University of Science and Technology and Univ. of Poitiers, Dec 2016.

[5] H. Deborah, N. Richard, and J. Y. Hardeberg. A comprehensive evaluation of spectral distance functions and metrics for hyperspectral image processing. Selected Topics in Applied Earth Observations and Remote Sensing, IEEE Journal of, 8(6):3224-3234, Jun 2015.

[6] H. Deborah, N. Richard, and J. Y. Hardeberg. Spectral ordering assessment using spectral median filters. In Mathematical Morphology and Its Applications to Signal and Image Processing, pages 387-397. Springer, 2015.

[7] S. Di Zenzo. A note on the gradient of a multi-image. In A. P. Inc., editor, Computer vision, Graphics, and Image Processing, volume 33, pages 116-125, 1986.

[8] A. N. Evans and X. U. Liu. A morphological gradient approach to color edge detection. IEEE Transactions on Image Processing, 15(6):1454-1463, 2006.

[9] F. C. Flores, A. M. Polidório, and R. d. A. Lotufo. The weighted gradient: A color image gradient applied to morphological segmentation. Journal of the Brazilian Computer Society, 11(3):53-63, 2005.

[10] F. A. Kruse, A. B. Lefkoff, J. W. Boardman, K. B. Heidebrecht, et al. The Spectral Image Processing System (SIPS)Interactive visualization and analysis of imaging spectrometer data. Remote Sens. Environ., 44(2-3):145-163, 1993.

[11] P. Lambert and L. Macaire. Filtering and segmentation: The specificity of colour images. In Colour in Graphics and Image Processing, 1st Intl. Conf. on, pages 57-71, Oct. 2000.

[12] K. Panetta, C. Gao, S. Agaian, and S. Nercessian. A new reference-based edge map quality measure. IEEE Transactions on Systems, Man, and Cybernetics: Systems, 46(11):1505-1517, Nov 2016.

[13] N. Richard, D. Helbert, C. Olivier, and M. Tamisier. Pseudo- divergence and bidimensional histogram of spectral differences for hyperspectral image processing. Journal of Imaging Science \& Technology, 60(5):50402-1-50402-13, 2016.

[14] J.-F. Rivest, P. Soille, and S. Beucher. Morphological gradients. Journal of Electronic Imaging, 2(4):326-336, 1993.

[15] G. Sapiro. Color snakes. Computer Vision and Image Understanding, 68(2):407-416, 1997.

[16] S. R. Vantaram, S. Piramanayagam, E. Saber, and D. Messinger. Spatial segmentation of multi/hyperspectral imagery by fusion of spectral-gradient-textural attributes. Journal of Applied Remote Sensing, 9(1):1-37, 2015.

[17] S. Velasco-Forero and J. Angulo. Advances in Low-Level Color Image Processing, chapter Vector Ordering and Multispectral Morphological Image Processing, pages 223-239. Springer Netherlands, Dordrecht, 2014.

[18] A. Wangenheim, R. Bertoldi, D. Abdala, et al. Color image segmentation using an enhanced gradient network method. Pattern Recognition Letters, 30(15):1404-1412, 2009.

[19] Q. Zhu. Efficient evaluations of edge connectivity and width uniformity. Image \& Vision Computing, 14(1):21-34, 1996.

\section{Author Biography}

Hilda Deborah received her BSc in computer science from the University of Indonesia (2010), her MSc from Erasmus Mundus Color in Informatics and Media Technology (2013), and her PhD in computer science from the University of Poitiers in France and NTNU in Norway (2016). She is currently a researcher at the Norwegian Colour \& Visual Computing Laboratory (Colourlab), NTNU. Her work focuses on the development of spectral mathematical morphology framework and its applications to the cultural heritage domain.

Noël Richard received his $P h D$ in signal processing from the University of Poitiers (France, 1993), where he is currently an Associate Professor. He coordinates axel of the NUMERIC research program, which is dedicated to digital services involving 19 laboratories, 180 researchers, regional and ERDF funds. He is also the technical chair of the CIE TC8-14 (spatio-chromatic complexity definition and assessment). His research activity is focused on metrological color and spectral image analysis and processing.

Jon Y. Hardeberg received his sivilingeniфr (MSc) degree in signal processing from the Norwegian Institute of Technology (Norway, 1995) and his PhD from Ecole Nationale Supérieure des Télécommunications (France, 1999). He is currently Professor of Colour Imaging at the Department of Computer Science at NTNU and a member of the Norwegian Colour \& Visual Computing Laboratory. His research interests include multispectral colour imaging, print and image quality, colorimetric device characterisation, colour management, and cultural heritage imaging.

Christine Fernandez-Maloigne graduated as a computer engineer (1986) and obtained her PhD degree in Image Processing (1989) from the University of Technology of Compiegne, France. She is currently a professor and vice-rector in charge of international relations at the University of Poitiers, director of MIRES, research federation. Her research activities focus on colour imaging, colour image and video filtering, segmentation, analysis and indexing, compression, watermarking and quality assessment. 